\section{Cardiovascular events were increased in patients with high cardiovascular risk who had albuminuria}

Gerstein HG, Mann JF, Yi Q, et al, for the HOPE Study Investigators. Albuminuria and risk of cardiovascular events, death, and heart failure in diabetic and nondiabetic individuals. JAMA 2001 Jul 25;286:421-6.

\section{QUESTION: Is any degree of albuminuria a risk factor for cardiovascular (CV) events in high risk patients with or without diabetes mellitus (DM)?}

\section{Design}

Cohort study of patients enrolled in the Heart Outcomes Prevention Evaluation (HOPE) study with a median 4.5 years of follow up.

\section{Setting}

Community and academic practices in Europe and North and South America.

\section{Participants}

9043 patients $\geqslant 55$ years of age who had a history of previous CV disease (either coronary artery disease, stroke, or peripheral vascular disease) or a history of DM plus $\geqslant 1$ other CV risk factor (total cholesterol concentration $>5.2 \mathrm{mmol} / \mathrm{l}$, high-density lipoprotein cholesterol concentration $\leqslant 0.9 \mathrm{mmol} / \mathrm{l}$, hypertension, known microalbuminuria, or current smoker). Exclusion criteria included dipstick-positive proteinuria or established diabetic nephropathy, other renal disease, hyperkalemia, congestive heart failure (CHF), low-ejection fraction, and hypersensitivity to vitamin $\mathrm{E}$ or angiotensinconverting enzyme inhibitors.

\section{Assessment of risk factors}

Baseline data were collected on age, sex, smoking status, hypertension, history of dyslipidaemia, DM status, abdominal obesity, and serum creatinine concentration. In patients with DM, duration of DM, use of oral glucose-lowering agents or insulin, and glycated haemoglobin concentrations were also assessed. Urinary creatinine and albumin concentrations were measured at baseline, first visit, and the end of the study in all patients; the serum creatinine concentration was measured at follow up visits in patients with DM.

\section{Main outcome measures}

CV events (myocardial infarction, stroke, or CV death), all-cause mortality, and admission to hospital for CHF.

\section{Main results}

Microalbuminuria was detected in 33\% of patients with $\mathrm{DM}$ and $15 \%$ of patients without DM, and it increased the risk for major CV events, all-cause mortality, and admitted to hospital for CHF (table). The risk increased with the albumin/creatinine ratio, beginning below the microalbuminuria cut point $(2 \mathrm{mg} / \mathrm{mmol})$. Similar relative risks (RRs) were seen for patients with or without DM and after adjustment for other CV risk factors.

\section{Conclusion}

Patients with increased cardiovascular risk, with or without diabetes mellitus, and any degree of albuminuria were at increased risk for major cardiovascular events. heart failure $(\mathrm{CHF})$ in all high risk patients

†Adjusted for randomisation to receive ramipril.

\section{COMMENTARY} patients with DM. marker of diffuse endothelial disease characteristic of early atherosclerosis. prescribe something more specific for albuminuria?
Sources of funding: Medical Research Council of Canada; Hoechst Marion Roussell; Astra; Natural Source Vitamin E Association; NEGMA; King Pharmaceuticals.

For correspondence: Dr H C Gerstein, Department of Medicine, McMaster University, Room 3V38, 1200 Main Street West, Hamilton, Ontario L8N 3Z5, Canada.Fax +1905 5214971

Incidence and relative risk (RR) of major cardiovascular $(C V)$ events (myocardial infarction, stroke, or cardiovascular death), all-cause mortality, and hospital admission for congestive

\begin{tabular}{llll}
$\begin{array}{l}\text { Outcomes at } \\
\text { median } 4.5 \text { years }\end{array}$ & $\begin{array}{l}\text { With } \\
\text { microalbuminuria }\end{array}$ & $\begin{array}{l}\text { Without } \\
\text { microalbuminuria }\end{array}$ & Adjusted RR (95\% Cl)† \\
Major CV events & $23 \%$ & $14 \%$ & $1.83(1.64$ to 2.05$)$ \\
\hline All-cause mortality & $18 \%$ & $9 \%$ & $2.09(1.84$ to 2.38$)$ \\
\hline $\begin{array}{l}\text { CHF hospital } \\
\text { admission }\end{array}$ & $7 \%$ & $2 \%$ & $3.23(2.54$ to 4.10$)$ \\
\hline
\end{tabular}

This large, international, multicentre, $\mathrm{CV}$ disease prevention trial - which found ramipril, but not vitamin E, protective - has clearly shown that the presence of albuminuria indicates increased risk for myocardial infarction, stroke, $\mathrm{CV}$ death, $\mathrm{CHF}$, and all-cause mortality. This increase was true in patients with or without diabetes and in those receiving ramipril or placebo. The increase in risk began even below the threshold concentration for microalbuminuria used to indicate incipient diabetic nephropathy in

The authors point out that many CV risk factors are associated with microalbuminuria, including smoking, dyslipidaemia, hypertension, hyperhomocystinemia, dietary protein, and markers of acute phase response. Although the authors adjusted for many of these factors in the analyses, further research is necessary to more clearly assess the independent CV risk signalled by albuminuria. More important, researchers need to clarify whether increasing albuminuria is a causal risk factor for $\mathrm{CV}$ disease or simply a

The steady increase in CV risk by deciles of albuminuria to a doubling of risk at the traditional concentration of microalbuminuria is so striking that clinicians may want to direct interventions to decrease albuminuria, but this approach may be premature. The study was based on one measure of albuminuria; thus, the individual variability in concentrations of albuminuria this low may be of concern. Cut points to stratify risk would have to be established. The clarity of the intervention must also be determined: should we intervene more aggressively in the areas of blood pressure, lipid concentrations, glucose intolerance, and smoking, which most physicians already try to do, or should we

Before clinicians begin measuring albuminuria for $\mathrm{CV}$ risk stratification, it will be necessary to assess whether albuminuria is a risk factor or just a marker for CV disease to determine the mechanism of any increased risk and to evaluate whether a specific intervention to lower albuminuria results in reduced CV risk. Meanwhile, clinicians should concentrate on reducing proven CV risk factors that have cleared these hurdles.

Donald A Smith, MD, MPH Mount Sinai Medical Center New York, New York, USA 